\title{
Efecto del protocolo de descongelación de semen sobre el porcentaje de preñez en bovinos lecheros
}

\author{
Bernardi, S.F.1,4; Di Prinzio, M.2,4; Maglione, D. ${ }^{3,4}$; Rinaudo, A. ${ }^{3,4}$; Marini, P.R..$^{2,4}$ \\ ${ }^{1}$ Histología I, ${ }^{2}$ Producción Bovinos Lecheros, ${ }^{3}$ Histología II, ${ }^{4}$ Centro Latinoamericano de Estudios de \\ Problemáticas Lecheras (CLEPL), Facultad Cs. Veterinarias, Universidad Nacional de Rosario, \\ Ruta 33 y Bv. Ov. Lagos, CP 2170, Casilda, Santa Fe, Argentina. Tel-Fax: 54-3464-422050. \\ E-mail: sbernard@unr.edu.ar
}

\begin{abstract}
Resumen
Bernardi, S.F.; Di Prinzio, M.; Maglione, D.; Rinaudo, A.; Marini, P.R.: Efecto del protocolo de descongelación de semen sobre el porcentaje de preñez en bovinos lecheros. Rev. vet. 26: 1, 27-32, 2015. Para analizar el efecto del protocolo de descongelación de semen sobre el porcentaje de preñez obtenido, se trabajó en tres tambos de Santa Fe (Argentina). Se evaluó el tiempo empleado en retirar la pajuela del termo de nitrógeno hasta depositarla en el baño para descongelar, la temperatura del agua y el lapso de inmersión de cada dosis para su descongelación, la duración de la carga de la dosis en la pistola y la concreción de la inseminación de cada hembra. El porcentaje de preñez se determinó a los 60 días post-inseminación por ecografía transrectal y la calidad del semen utilizado se analizó mediante sistema CASA (SpermVision, Minitub, Alemania). El protocolo de descongelación no tuvo impacto sobre la preñez puesto que, en cada establecimiento, se cuidaron cada uno de sus pasos de manera exhaustiva hasta que la dosis seminal fue inseminada. Por otra parte, el estado reproductivo varió significativamente en relación a la persona que realizó la inseminación y al momento del año en que se desarrolló la actividad. Pese a que se imponen más estudios para profundizar el tema, puede adelantarse que el mayor impacto sobre el porcentaje de preñez residió en factores externos al manejo de las pajuelas y a la calidad del semen.
\end{abstract}

Palabras clave: bovino lechero, inseminación artificial, manipulación seminal, calidad del semen descongelado, tasa de preñez.

\begin{abstract}
Bernardi, S.F.; Di Prinzio, M.; Maglione, D.; Rinaudo, A.; Marini, P.R.: Effect of unfreezing semen protocol on the dairy cows' pregnancy rate. Rev. vet. 26: 1, 27-32, 2015. In order to analyze the effect of the protocol of semen thawing on the percentage of final pregnancy, a trial was carried out in three farms in Santa Fe (Argentina). It was evaluated the time spent in taking out the straw from the nitrogen thermus till placing it in the thawing bath, as well as the temperature of the water together with the immersion time of each dose till its thawing, the length each dose remained in the inseminating gun and the performing of the insemination in each cow. The percentage of pregnancy was determined 60 days after the insemination through a transrectal ultrasound and the quality of the semen by means of the CASA system (Sperm Vision, Minitub, Germany). The protocol of thawing did not have an impact on pregnancy since every step was thoroughly controlled till the semen dose was inseminated. On the other side, the reproductive state varied significantly considering the operator performing the insemination and the time of the year. Even though more studies need to be carried out in order to go further into the topic, it can be anticipated that the higher impact on the percentage of pregnancy lays in factors other than the straw handling and the quality of semen.
\end{abstract}

Key words: dairy cattle, artificial insemination, semen manipulation, thawed semen quality, pregnancy rate. 


\section{INTRODUCCIÓN}

Cuando se inseminan hembras fértiles con una condición corporal adecuada, el programa de inseminación artificial resultará exitoso si se cuenta con un eficiente sistema de detección de celos, si se utiliza semen de buena calidad y si la técnica es ejecutada por inseminadores avezados. Sin embargo al seleccionar un toro, se tiene en cuenta su genética y su fertilidad medida como tasa de no retorno o porcentaje de preñez y sólo en algunas ocasiones, por la calidad del semen en sí misma. Muchos autores trabajaron sobre las distintas pruebas que dan cuenta de la calidad seminal, basándose en parámetros diversos como motilidad, integridad del acrosoma, funcionalidad de la membrana celular e integridad del ADN, entre otros $1,4,10,14$.

Sin embargo, no son tan numerosas las investigaciones realizadas sobre el efecto "ambiente", que influye sobre la calidad final del semen que será inseminado. Esto se refiere a las condiciones de almacenamiento de las dosis y al protocolo de descongelación utilizado, que en general no son tenidos en cuenta al momento de evaluar el resultado final de la inseminación realizada ${ }^{5}$. El proceso de descongelación del semen es de extremo cuidado, por un lado porque actualmente se comercializa en pajuelas de 0,50 o $0,25 \mathrm{~cm}^{3}$, envases plásticos cuya relación superficie-volumen es muy elevado, lo que las torna altamente sensibles a cualquier cambio del protocolo. Únicamente cumpliendo de manera estricta el protocolo, podrá garantizarse que el semen inseminado conserve la máxima calidad respecto de la original.

El tiempo en que se produce la descongelación depende del tamaño y forma del recipiente en que se envasó el semen, de su composición, del medio de descongelación, de la temperatura utilizada y del lapso que se mantenga a dicha temperatura. Estudios previos recomiendan efectuar una descongelación rápida utilizando baños de agua tibios $\left(35-37^{\circ} \mathrm{C}\right)$, por cortos períodos de tiempo estrictamente controlados (30-35 segundos). El tiempo de inmersión de las pajuelas debe ser el suficiente para que el semen pueda ir incrementando progresivamente la temperatura hasta llegar a una equiparable a la del útero de la vaca $\left(38,5^{\circ} \mathrm{C}\right)$.

Este incremento progresivo de la temperatura durante el protocolo de descongelación de las dosis evita el shock térmico que provocaría la muerte de los espermatozoides. Un manejo adecuado del semen congelado mejoraría los porcentajes de fertilidad obtenidos por esta técnica, especialmente si se tienen en cuenta factores como experiencia y habilidad del inseminador, correcta detección del celo, adecuado momento de inseminación durante el estro y depósito del semen en el sitio más adecuado del útero, entre otros ${ }^{8}$.

El presente trabajo tuvo como objetivo analizar el protocolo de descongelación utilizado en distintos tambos del sur de la Provincia de Santa Fe, la calidad del semen y el porcentaje de preñez obtenido.

\section{MATERIAL Y MÉTODOS}

Lugares de trabajo, animales, maniobras efectuadas. Se trabajó en tres establecimientos lecheros del sur de la Provincia de Santa Fe, Argentina. La alimentación se basó en praderas de alfalfa con distintos niveles de suplementación (balanceado comercial, silo de maíz y rollos de alfalfa). Se utilizaron 236 vacas lecheras Holstein de primer parto clínicamente sanas y con estado corporal homogéneo (3,25 en la escala 1-5). En el momento de realizar las inseminaciones se controló, en forma estricta y cronometrada cada paso del protocolo de descongelación de las pajuelas utilizadas en la inseminación, también se tomó registro del toro utilizado y del inseminador actuante a fin de poder dilucidar sus efectos sobre la tasa de preñez. A los 60 días posteriores a la inseminación se realizó la revisión de los animales por tacto y/o ecografía transrectal para constatar el estado reproductivo de cada vaca: preñada o vacía.

Protocolo de sincronización. Día 0: inserción del DIB y aplicación de $2 \mathrm{mg}$ de benzoato de estradiol. Día 7: retiro del dispositivo. Aplicación de prostaglandina y cipionato de estradiol. Inseminación artificial a tiempo fijo (IATF) a 56 horas del día 7.

Protocolo de descongelación de las dosis seminales. Se cronometró el tiempo que llevó retirar la dosis desde el termo de nitrógeno hasta depositarla en el termo de descongelación; el tiempo de inmersión de cada pajuela en dicho recipiente y el tiempo transcurrido desde que se retiró la dosis del termo caliente hasta que, una vez cargada, se depositó en el tracto reproductor de la hembra.

Análisis de la calidad del semen descongelado. Las muestras analizadas correspondieron a pajuelas de cada toro utilizado en las inseminaciones que formaron parte de este estudio; fueron 8 toros en total y se utilizaron 3 dosis por cada uno de ellos. Las mismas se descongelaron previamente y se volcaron a un tubo de vidrio del cual se tomó la alícuota necesaria para realizar los exámenes previstos. Mediante el uso de un sistema de análisis de semen computarizado se calculó la motilidad, progresión y velocidad de los espermatozoides (SpermVision, Minitüb, Alemania) utilizando cámara Leja de $2 \mu \mathrm{l}$ y analizando 10 campos microscópicos por muestra. Este análisis se realizó en la Cooperativa de Inseminación Artificial de Venado Tuerto (CIAVT, Santa Fe, Argentina). Los parámetros CASA (Computer Assisted Semen Analysis) fueron los siguientes:

- porcentaje de espermatozoides progresivos (PRO): porcentaje de células que siendo móviles, describen un desplazamiento hacia delante.

- velocidad curvolineal (VCL): velocidad calculada sobre el camino real del espermatozoide entre dos puntos de su trayectoria, expresada en micrones por 
Tabla 1. Etapas del protocolo de descongelación por establecimiento lechero.

\begin{tabular}{lccccc}
\hline tambo & $\mathrm{n}$ & $\begin{array}{c}\text { tiempo en retirar pajuela } \\
\text { del nitrógeno }(\mathrm{s})\end{array}$ & $\begin{array}{c}\text { temperatura de } \\
\text { descongelación }\left({ }^{\circ} \mathrm{C}\right)\end{array}$ & $\begin{array}{c}\text { tiempo en baño de } \\
\text { descongelación }(\mathrm{s})\end{array}$ & $\begin{array}{c}\text { tiempo desde carga de } \\
\text { pajuela hasta inseminar }(\mathrm{s})\end{array}$ \\
\hline E1 & 112 & $3,83 \pm 0,16 \mathrm{a}$ & $34,61 \pm 0,06 \mathrm{~b}$ & $66,74 \pm 6,84 \mathrm{~b}$ & $66,88 \pm 4,19 \mathrm{c}$ \\
$\mathrm{E} 2$ & 76 & $3,46 \pm 0,12 \mathrm{a}$ & $34,43 \pm 0,13 \mathrm{~b}$ & $97,17 \pm 9,79 \mathrm{a}$ & $95,89 \pm 8,54 \mathrm{~b}$ \\
$\mathrm{E} 3$ & 48 & $3,48 \pm 1,17 \mathrm{a}$ & $34,98 \pm 0,12 \mathrm{a}$ & $55,64 \pm 1,07 \mathrm{~b}$ & $135,79 \pm 13,85 \mathrm{a}$ \\
\hline
\end{tabular}

E: establecimiento. Valores en $\bar{x} \pm$ EE. Letras distintas dentro de una columna indican diferencias significativas $(p<0,05)$.

segundo; describe la motilidad real de los espermatozoides.

- velocidad lineal (VSL): velocidad del espermatozoide sobre una línea recta entre dos puntos medida en micrones por segundo.

- velocidad promedio en su trayectoria (VAP): velocidad promedio del espermatozoide sobre el camino real, expresada en micrones por segundo.

- linealidad $(\mathrm{LIN}=(\mathrm{VSL} / \mathrm{VCL}) * 100)$ : desviación de la velocidad del camino real a la velocidad en línea recta expresada en porcentaje.

- rectitud $(\mathrm{STR}=(\mathrm{VSL} / \mathrm{VAP}) * 100)$ : desviación de la velocidad promedio a la velocidad en línea recta expresada en porcentaje.

- amplitud lateral de la cabeza (ALH): distancia promedio del desplazamiento de la cabeza desde la posición media del camino, expresada en micrones.

- frecuencia de batido (BCF): frecuencia de corte de los movimientos laterales de la cabeza del espermatozoide, expresada en Hertz (Hz).

Evaluación del acrosoma. Se colocó una gota de semen sobre un portaobjetos y al lado una gota similar de glutaraldehído al $0,2 \%$ diluido con buffer de fosfato salino. Se realizaron tres lecturas por cada pajuela descongelada utilizando un microscopio de contraste de fase (1000X), contando 100 espermatozoides en cada extendido. Para ello la muestra de semen se fijó con glutaraldehído al $2 \%$. Se prestó preferencial atención a defectos como la falta total del capuchón acrosómico o su pérdida en distintas proporciones, expresándose la anomalía como porcentaje de acrosomas dañados (AD).

Análisis estadístico. Se obtuvieron media aritmética y error estándar de todas las variables medidas. Se verificó la existencia de diferencias significativas entre los protocolos de descongelación, mediante la aplicación de análisis de la variancia a un criterio de clasificación y pruebas de comparaciones múltiples HSD de Tukey-Kramer. Se calcularon coeficientes de Pearson a los efectos de establecer relaciones entre las variables estudiadas; además se utilizó una regresión logística para conocer el peso de distintas variables sobre el estado reproductivo final de los animales (Test Wald). Todos los análisis estadísticos fueron realizados utilizando el programa JMP en su versión 5.0 para Windows (JMP $\left.{ }^{\circledR}, 2003\right)$.

\section{RESULTADOS}

Control de la manipulación y descongelación de la dosis hasta la inseminación. En el momento de realizar la inseminación se controlaron, para cada animal, cuatro etapas referidas al acondicionamiento del semen que sería depositado en el útero: tiempo empleado en retirar la pajuela del termo de nitrógeno líquido hasta depositarla en el baño caliente; temperatura del agua utilizada en dicho baño; tiempo de inmersión de cada dosis en el agua para su descongelación y tiempo utilizado para retirar la pajuela, cargarla en la pistola y depositar el semen en el tracto reproductivo de la hembra.

Los resultados se muestran en la Tabla 1, destacándose que hubo diferencias estadísticamente significativas en la temperatura del agua y tiempo en el baño de descongelación, así como en el tiempo empleado para cargar la pajuela e inseminar. Al calcular el coeficiente de regresión entre dichas variables, resultó que ningu-

Tabla 2. Parámetros de calidad del semen de cada toro.

\begin{tabular}{lcccccccc}
\hline parámetro & S1 & S2 & S3 & S4 & S5 & S6 & S7 & S8 \\
\hline \multirow{2}{*}{ PRO } & 57,62 & 59,85 & 55,50 & 59,30 & 56,21 & 57,90 & 58,80 & 55,70 \\
& $\pm 1,01 \mathrm{a}$ & $\pm 1,06 \mathrm{a}$ & $\pm 0,94 \mathrm{a}$ & $\pm 0,68 \mathrm{a}$ & $\pm 0,36 \mathrm{a}$ & $\pm 0,90 \mathrm{a}$ & $\pm 1,01 \mathrm{a}$ & $\pm 0,57 \mathrm{a}$ \\
VCL & 193,22 & 215,25 & 183,60 & 200,50 & 180,80 & 191,67 & 201,10 & 198,50 \\
& $\pm 2,54 \mathrm{a}$ & $\pm 1,02 \mathrm{a}$ & $\pm 1,50 \mathrm{a}$ & $\pm 1,30 \mathrm{a}$ & $\pm 0,80 \mathrm{a}$ & $\pm 0,83 \mathrm{a}$ & $\pm 0,91 \mathrm{a}$ & $\pm 0,91 \mathrm{a}$ \\
VAP & 100,50 & 100,25 & 102,03 & 112,50 & 99,60 & 100,90 & 101,12 & 104,06 \\
& $\pm 2,81 \mathrm{a}$ & $\pm 1,00 \mathrm{a}$ & $\pm 0,95 \mathrm{a}$ & $\pm 0,95 \mathrm{a}$ & $\pm 1,45 \mathrm{a}$ & $\pm 0,62 \mathrm{a}$ & $\pm 0,16 \mathrm{a}$ & $\pm 1,33 \mathrm{a}$ \\
\multirow{2}{*}{ VSL } & 90,8 & 88,06 & 90,10 & 92,70 & 91,80 & 90,80 & 87,60 & 91,90 \\
& $\pm 0,75 \mathrm{a}$ & $\pm 0,81 \mathrm{a}$ & $\pm 1,01 \mathrm{a}$ & $\pm 0,68 \mathrm{a}$ & $\pm 0,25 \mathrm{a}$ & $\pm 1,10 \mathrm{a}$ & $\pm 0,22 \mathrm{a}$ & $\pm 0,64 \mathrm{a}$ \\
\multirow{2}{*}{ LIN } & 0,65 & 0,50 & 0,42 & 0,49 & 0,56 & 0,47 & 0,53 & 0,61 \\
& $\pm 0,00 \mathrm{a}$ & $\pm 0,01 \mathrm{a}$ & $\pm 0,01 \mathrm{a}$ & $\pm 0,01 \mathrm{a}$ & $\pm 0,01 \mathrm{a}$ & $\pm 0,00 \mathrm{a}$ & $\pm 0,00 \mathrm{a}$ & $\pm 0,01 \mathrm{a}$ \\
\multirow{2}{*}{$\mathrm{STR}$} & 0,83 & 0,91 & 0,86 & 0,86 & 0,88 & 0,76 & 0,80 & 0,80 \\
& $\pm 0,00 \mathrm{~b}$ & $\pm 0,01 \mathrm{a}$ & $\pm 0,01 \mathrm{a}$ & $\pm 0,00 \mathrm{a}$ & $\pm 0,00 \mathrm{a}$ & $\pm 0,00 \mathrm{~b}$ & $\pm 0,01 \mathrm{a}$ & $\pm 0,01 \mathrm{a}$ \\
\multirow{2}{*}{ BCF } & 31,50 & 30,50 & 29,75 & 30,30 & 32,24 & 30,15 & 29,51 & 30,67 \\
& $\pm 0,43 \mathrm{a}$ & $\pm 0,85 \mathrm{a}$ & $\pm 1,05 \mathrm{a}$ & $\pm 0,42 \mathrm{a}$ & $\pm 0,22 \mathrm{a}$ & $\pm 0,93 \mathrm{a}$ & $\pm 0,88 \mathrm{a}$ & $\pm 1,02 \mathrm{a}$ \\
\multirow{2}{*}{$\mathrm{ALH}$} & 5,04 & 5,10 & 5,00 & 5,61 & 4,86 & 4,22 & 5,59 & 5,59 \\
& $\pm 0,09 \mathrm{a}$ & $\pm 0,22 \mathrm{a}$ & $\pm 0,20 \mathrm{a}$ & $\pm 0,27 \mathrm{a}$ & $\pm 0,61 \mathrm{a}$ & $\pm 0,90 \mathrm{a}$ & $\pm 0,40 \mathrm{a}$ & $\pm 0,40 \mathrm{a}$ \\
\multirow{2}{*}{ AD } & 20,15 & 18,50 & 21,60 & 19,27 & 22,00 & 19,80 & 21,00 & 20,00 \\
& $\pm 0,18 \mathrm{a}$ & $\pm 1,13 \mathrm{a}$ & $\pm 0,57 \mathrm{a}$ & $\pm 0,50 \mathrm{a}$ & $\pm 1,00 \mathrm{a}$ & $\pm 0,60 \mathrm{a}$ & $\pm 0,35 \mathrm{a}$ & $\pm 0,69 \mathrm{a}$ \\
\hline
\end{tabular}

parámetro: ver texto. S: semen. Valores en $\overline{\mathrm{X}} \pm \mathrm{EE}$. Diferentes letras en la misma fila indican diferencias significativas $(\mathrm{p}<0,05)$ 
na de las diferencias encontradas en los pasos involucrados en la manipulación y descongelación del semen tuvo relación con el porcentaje de preñez alcanzado.

Calidad del semen. Los análisis realizados demostraron que la calidad del semen utilizado en los tres establecimientos fue similar, puesto que no se encontraron diferencias estadísticamente significativas para ninguno de los parámetros evaluados (Tabla 2). El coeficiente de correlación entre el porcentaje de preñez de cada toro con el porcentaje de espermatozoides móviles progresivos $(\mathrm{r}=-0,2801)$ y con la linealidad $(\mathrm{r}$ $=0,52508$ ) resultó bajo, evidenciando ausencia de relación entre estas variables.

Estado reproductivo. El porcentaje de preñez obtenido en la IATF fue similar para los tres tambos analizados, promediando 52,59\% (Tabla 3). Dado que las actividades se desarrollaron en distintos meses del año,

Tabla 3. Porcentaje de vacas preñadas (P) y vacías (V) por establecimiento (E).

\begin{tabular}{lccc}
\hline tambo & $\% \mathrm{P}$ & $\% \mathrm{~V}$ & $\%$ total \\
\hline E1 & 42,86 & 57,14 & 100 \\
E2 & 56,58 & 43,42 & 100 \\
E3 & 58,33 & 41,67 & 100 \\
$\overline{\mathbf{x}}$ & 52,59 & 47,41 & - \\
\hline Test & & ChiSquare & Prob $>$ ChiSq \\
Likelihood Ratio & & 4,936 & 0,0848 \\
Pearson & & 4,918 & 0,0855 \\
\hline
\end{tabular}

Tabla 4. Porcentaje de vacas preñadas $(\mathrm{P})$ y vacías $(\mathrm{V})$ por estación del año.

\begin{tabular}{lcc}
\hline estación & $\% \mathrm{P}$ & $\% \mathrm{~V}$ \\
\hline invierno & 27,73 & 23,08 \\
otoño & 39,50 & 56,41 \\
primavera & 32,77 & 20,51 \\
\% total & 100 & 100 \\
\hline Test & ChiSquare & Prob $>$ ChiSq \\
Likelihood Ratio & 7,400 & 0,0247 \\
Pearson & 7,350 & 0,0254 \\
\hline
\end{tabular}

Tabla 5. Porcentaje de hembras preñadas $(\mathrm{P})$ y vacías (V) según el inseminador actuante.

\begin{tabular}{lccc}
\hline inseminador & $\% \mathrm{P}$ & $\% \mathrm{~V}$ & total \\
\hline $\mathrm{A}$ & 42,86 & 57,14 & 100 \\
$\mathrm{~B}$ & 67,86 & 32,14 & 100 \\
$\mathrm{C}$ & 75,00 & 25,00 & 100 \\
$\mathrm{D}$ & 56,82 & 43,18 & 100 \\
$\mathrm{E}$ & 51,61 & 48,39 & 100 \\
$\mathrm{~F}$ & 44,44 & 55,56 & 100 \\
$\mathrm{G}$ & 50,00 & 50,00 & 100 \\
\hline Test & ChiSquare & Prob $>$ ChiSq & RSquare (U) \\
Likelihood Ratio & 13,337 & 0,0204 & 0,0501 \\
Pearson & 11,656 & 0,0398 & \\
\hline
\end{tabular}

atravesando tres de las cuatro estaciones (primavera, otoño e invierno), en la Tabla 4 se muestran los resultados de preñez obtenidos según la época en que se efectuó la inseminación, resultando ligeramente superiores en las vacas inseminadas durante el otoño.

Inseminador. En cada inseminación se identificó el inseminador actuante, registrándose los resultados obtenidos por cada uno (preñadas/vacías) en la Tabla 5. Al evaluar los porcentajes de hembras preñadas y vacías a los 60 días post-inseminación, se observaron diferencias significativas entre quienes realizaron la maniobra.

De todas las variables independientes estudiadas para el protocolo de descongelación (regresión logística *ProbChiSq-Test y Wald), la calidad del semen, el inseminador actuante y la fecha de realización de la inseminación, las que tuvieron un mayor peso a fin de definir la preñez en una hembra fueron: la época del año en la que se efectuó la inseminación $\left(0,0001^{*}\right)$, el tiempo empleado en sumergir la dosis en agua caliente para su descongelación $\left(0,0163^{*}\right)$ y el inseminador actuante $(0.0248 *)$.

\section{DISCUSIÓN}

En la inseminación artificial, el proceso de descongelación de una dosis es un momento crítico porque para conservar la máxima fertilidad del semen es necesario que se respeten cada uno de los pasos del proceso y que los operarios se preparen debidamente a tal fin ${ }^{5,6}$. En los establecimientos muestreados para este trabajo, en general, el protocolo de descongelación fue el adecuado. Si bien la temperatura utilizada para la descongelación de las dosis fue diferente en algunos tambos, el promedio se mantuvo dentro de los valores recomendados y usualmente elegidos por los inseminadores, por tanto estas diferencias no deben ser consideradas como una práctica adversa en lo que respecta a la calidad seminal.

En relación a las diferencias significativas encontradas en el tiempo empleado para descongelar la pajuela por inmersión de la misma en agua atemperada, se observó que las mismas surgieron a causa de que los operarios retiraban del nitrógeno de a dos, tres o más dosis por vez. Así, en una de ellas se respetó exactamente el tiempo estipulado correspondiente a la temperatura del agua elegida, mientras que en la o las restantes el tiempo de inmersión se extendió más allá de lo aconsejado.

Numerosos autores han demostrado que el control de la temperatura y el tiempo de exposición del baño de descongelación resultaron de vital importancia para mantener intactas las características estructurales y funcionales en la mayor cantidad posible de espermatozoides. Las temperaturas extremas $\left(21^{\circ} \mathrm{C}\right.$ por 5 minutos, $75^{\circ} \mathrm{C}$ por 9 segundos y $95^{\circ} \mathrm{C}$ por 7 segundos), tuvieron un efecto dañoso significativo sobre los espermatozoi- 
des móviles progresivos, la velocidad, la distancia recorrida a lo largo de la trayectoria de desplazamiento y sobre el porcentaje de espermios con una membrana funcionalmente activa.

Sin embargo, las temperaturas más elevadas permitieron reducir significativamente el porcentaje de espermatozoides con acrosomas dañados, característica comprometida directamente con el proceso de penetración al oocito ${ }^{2,16,18}$. Las altas temperaturas en general inciden sobre la calidad del semen obtenido al descongelar; sin embargo, controlar tales temperaturas a campo y además teniendo en cuenta que los tiempos de inmersión son muy breves, resulta inviable en este tipo de protocolo ${ }^{5}$. Los protocolos con temperaturas intermedias $\left(35^{\circ} \mathrm{C}, 37^{\circ} \mathrm{C}\right.$ y $\left.40^{\circ} \mathrm{C}\right)$ resultaron en una calidad superadora cuando se complementaron con tiempos no menores a 30 segundos y no mayores a un minuto.

Otros estudios, compararon la fertilidad obtenida al utilizar semen descongelado a $37^{\circ} \mathrm{C}$ con distintos tiempos de inmersión en el baño; los mejores resultados se obtuvieron al extender el tiempo en que la pajuela se sumerge en el agua caliente entre 30 y 60 segundos ${ }^{5,15}$. El tiempo de exposición de la pajuela en el agua caliente fue también motivo de estudio por distintos autores; así se demostró que la exposición de semen por más de un minuto en el agua caliente para su descongelación provocó la disminución del porcentaje de espermatozoides motiles y progresivos; y una vez descongelada, debe ser inseminada inmediatamente cuidando que no sufra cambios bruscos de temperatura.

Desaciertos en el tiempo o en la temperatura a la que se expone la dosis luego de ser descongelada, afectaron parámetros que hacen referencia a la funcionalidad de la membrana celular y del acrosoma, motilidad, progresión y frecuencia de batido de los espermatozoides $^{7,11}$. También se observaron variaciones significativas en el tiempo empleado desde la carga de la dosis en la pistola hasta efectivizar la inseminación. Esto estuvo en relación a formas de trabajo y prácticas propias de cada lugar y persona, por ejemplo, la demora en enhebrar y alcanzar el lugar adecuado para depositar los espermatozoides.

Las dosis seminales utilizadas en este trabajo cumplieron con los estándares de calidad requeridos, entendiendo por calidad respecto de una dosis, una concentración mínima de treinta millones de espermatozoides totales con un $30 \%$ de motilidad progresiva luego de su descongelación, o lo que es lo mismo un total de 9 a 10 millones de espermatozoides motiles progresivos. Es decir, que en base a los resultados aquí obtenidos, el porcentaje de preñez a los 60 días post-inseminación no estuvo en relación con la cantidad de espermatozoides motiles progresivos o con la linealidad, que es un parámetro que relaciona la desviación de la velocidad del camino real respecto a la velocidad en línea recta expresada en porcentaje.

Cabe señalar que los resultados expuestos anteriormente coinciden con los obtenidos por otros autores, los que al inseminar con dosis que contenían 6,10 y 12 millones de espermatozoides con motilidad progresiva, no encontraron diferencias significativas en el porcentaje de preñez a la $\operatorname{IATF}^{9}$. Sin embargo, otros sostienen que el número de células motiles, y secundariamente la linealidad, son parámetros que pueden ser utilizados para predecir la fertilidad de un toro, puesto que se encontró una correlación significativa entre el porcentaje de motiles progresivos y el porcentaje de fertilización ${ }^{3}$. En el presente trabajo, al evaluar el manejo de las dosis en el proceso de descongelación, pudo afirmarse que esta práctica se cumplió respetando las pautas estipuladas; de este modo no sería un factor que esté ejerciendo un impacto negativo sobre el porcentaje de preñez, al menos en esta oportunidad.

El porcentaje de preñez se modificó en relación a la fecha de inseminación, observándose una mayor tasa durante el otoño. En la bibliografía no se hallaron registros de un mayor índice de preñez en los meses de esta estación respecto de otros. La estacionalidad de los trabajos reproductivos en el tambo obedecen a causas diversas. En general, la concentración de los servicios en determinadas épocas del año, ya sea que se trabaje con servicio natural o con inseminación artificial, tiene por objeto hacer coincidir las pariciones con la mejor época de producción de pasto o con la mayor disponibilidad de alimento, situación favorable para las distintas categorías de animales. Otra finalidad podría estar relacionada con la organización de las actividades, concentrando éstas en determinados períodos para que el personal pueda prestarles mayor atención a las más importantes, como ser detección de celos y atención de partos, entre otras ${ }^{12}$.

El porcentaje de preñez también estuvo influenciado por el operador que realizó la inseminación, variando entre 44 y $75 \%$. Estos resultados concuerdan con investigaciones que reportaron variaciones de preñez entre 44,9 y $71,8 \%{ }^{19}$. Una situación similar, aunque con un rango de variación más amplio que el aquí encontrado, fue comunicado por autores que registraron una variación entre 23,5 y 77,7\%, asociándose los mejores resultados con las personas de mayor experiencia y formación técnica específica que realizaron las tareas a campo y de inseminación ${ }^{13,17}$.

Agradecimientos. A los propietarios, tamberos y personal de los establecimientos donde se realizó el trabajo, por su apoyo y colaboración. A la Cooperativa de Inseminación Artificial de Venado Tuerto (CIAVT) por permitirnos realizar el análisis computarizado de semen y poner a nuestra disposición su laboratorio y personal técnico.

\section{REFERENCIAS}

1. Anzar M, Rasul Z, Ahmed TA, Ahmad N. 2010. Response of buffalo spermatozoa to low temperatures during cryopreservation. Reprod Fertil Dev 22: 871-880.

2. Bacinoglu S, Tas M, Cirit Ü, Özdas ÖB, Aka K. 2008. The potential fertility estimation capacity of the hypoos- 
motic swelling test, the thermal stress test and a modified cervical mucus penetration test in the bovine. Anim Reprod Sci 104: 38-46.

3. Brogliatti GM. 2010. El impacto de calidad seminal en los programas de IATF. On line: http://www.abspecplan. com.br/upload/library/Impacto_seminal.pdf

4. Cormier N, Sirrd M, Bailey J. 1997. Premature capacitation of bovine spermatozoa is initiated by cryopreservation. $J$ Androl 18: 461-468.

5. Decuadro-Hansen G. 2010. Manejo del semen en un programa de IATF: aspectos críticos para preservar la fertilidad (conferencia). Resúmenes $5^{\circ}$ Jornadas TAURUS de Reproducción Bovina, Campus Nuestra Señora del Pilar, Universidad del Salvador, Buenos Aires, Argentina.

6. Decuadro-Hansen G. 2012. Factores que influyen en la congelación del semen de toros. On line: http://www. engormix.com/MA-ganaderia-carne/genetica/articulos/f actores-influyen-congelacion-semen-103-t4305/p0.htm.

7. Dejarnette JM, Barnes DA, Marshall CE. 2000. Effects of pre and post- thaws thermal insults on viability characteristics of cryopreserved bovine semen. Theriogenology 53: 1225-1238.

8. De los Reyes M. 2012. Manejo del semen congelado para inseminación artificial. Revista online de Reproducción Animal, http://www.reproduccionanimal.net/v02/manejodel-semen-congelado-para-inseminacion-artificial/.

9. Echeverría S, Zapiola A, Cabodevilla J, Callejas S. 2006. Efecto del momento de administrar el benzoato de estradiol al finalizar un tratamiento con progesterona y del número de espermatozoides con motilidad progresiva sobre el porcentaje de preñez a la IATF. Rev Arg Prod Anim 26 (Supl.1): 288-289.

10. Foote R. 2003. Fertility estimation: a review of past experience and future prospects. Anim Reprod Sci 75: 119-139.

11. García-Guerra A, Etcheverry MP, Larraburu G, Brogliatti GM. 2008. Efecto de la exposición de semen congelado al ambiente por distintos tiempos evaluado mediante el sistema CASA. Memorias Iras Jornadas Internacionales del INITRA, Fac. Cs. Vet. UBA. InVet 10 (2): 169.
12. Glauber CE. 2007. Manejo reproductivo en el rodeo bovino lechero: propuestas y reflexiones. On line at: http:// www.engormix.com/MA-ganaderia-leche/genetica/art iculos/manejo-reproductivo-rodeo-bovino-t1415/103-p0. htm

13. Mazzarri G, Fuenmayor C. 1973. Comportamiento y eficiencia reproductiva en vacas lecheras (conferencia). Memorias Seminario sobre Producción de Leche en Venezuela, p.153-170. Citado en Zoot Trop (1995) 13: 129-149.

14. Muiño R, Fernández M, Areán H, Viana JL, López M, Fernández A, Peña AI. 2005. Nuevas tecnologías aplicadas al procesado y evaluación del semen bovino en centros de inseminación artificial. Rev ITEA 101: 175-191.

15. Pace MM, Sullivan JJ, Elliott FI, Graham EF, Coylter GH. 1981. Effects of thawing temperature, number spermatozoa and spermatozoa quality on fertility of bovine spermatozoa packaged in $0.5 \mathrm{ml}$ French straws. J Anim Sci 53: 693-701.

16. Rodríquez DL, Berndtson WE, Ennew BD, Pickett BW. 1975. Effect of rates of freezing, thawing and level of glicerol on the survival of bovine spermatozoa in straws. J Anim Sci 41: 129-133.

17. Rodríguez-Hernández T, Espinoza J, Verde O. 1995. Efecto del momento de inseminación artificial, masaje clitórico, temperatura rectal y otros factores sobre la preñez en bovinos. Zootecnia Trop 13: 129-149.

18. Rubio-Guillén J, Quintero-Moreno A, González Villalobos D. 2009. Efecto de la criopreservación sobre la integridad de la membrana plasmática y acrosomal de espermatozoides de toro. Rev Cientif FCV-LUZ 19: 382-389.

19. Shannon P. 1965. Contribution of binomial variation, technician, herds and cows to observation between cow variance of conception rate. J Dairy Sci 48: 85-89. 